\title{
Bladder cancer stage and mortality: urban vs. rural residency
}

\author{
Marina Deuker ${ }^{1,2}(1) \cdot$ L. Franziska Stolzenbach ${ }^{2,3} \cdot$ Claudia Collà Ruvolo $^{2,4} \cdot$ Luigi Nocera $^{2,5} \cdot$ Zhe Tian $^{2}$. \\ Frederik C. Roos ${ }^{1} \cdot$ Andreas Becker $^{1} \cdot$ Luis A. Kluth $^{1}$ - Derya Tilki ${ }^{3}$. Shahrokh F. Shariat ${ }^{6,7,8}$. Fred Saad ${ }^{2}$. \\ Felix K.H. Chun ${ }^{1}$ - Pierre I. Karakiewicz ${ }^{2}$
}

Received: 16 August 2020 / Accepted: 6 November 2020 / Published online: 23 November 2020

(c) The Author(s) 2020

\begin{abstract}
Objective Relative to urban populations, rural patients may have more limited access to care, which may undermine timely bladder cancer (BCa) diagnosis and even survival.

Methods We tested the effect of residency status (rural areas [RA $<2500$ inhabitants] vs. urban clusters [UC $\geq 2500$ inhabitants] vs. urbanized areas [UA, $\geq 50,000$ inhabitants]) on $\mathrm{BCa}$ stage at presentation, as well as on cancer-specific mortality (CSM) and other cause mortality (OCM), according to the US Census Bureau definition. Multivariate competing risks regression (CRR) models were fitted after matching of RA or UC with UA in stage-stratified analyses.

Results Of 222,330 patients, 3496 (1.6\%) resided in RA, 25,462 (11.5\%) in UC and 193,372 (87\%) in UA. Age, tumor stage, radical cystectomy rates or chemotherapy use were comparable between RA, UC and UA (all $p>0.05$ ). At 10 years, RA was associated with highest OCM followed by UC and UA (30.9\% vs. $27.7 \%$ vs. $25.6 \%, p<0.01)$. Similarly, CSM was also marginally higher in RA or UC vs. UA $(20.0 \%$ vs. $20.1 \%$ vs. $18.8 \%, p=0.01)$. In stage-stratified, fully matched CRR analyses, increased OCM and CSM only applied to stage T1 BCa patients.

Conclusion We did not observe meaningful differences in access to treatment or stage distribution, according to residency status. However, RA and to a lesser extent UC residency status, were associated with higher OCM and marginally higher CSM in T1N0M0 patients. This observation should be further validated or refuted in additional epidemiological investigations.
\end{abstract}

Keywords Bladder cancer $\cdot$ Social differences $\cdot$ Stage at presentation $\cdot$ Geographical disparities $\cdot$ Treatment rates

Marina Deuker

Marina.Deuker@kgu.de

1 Department of Urology, University Hospital Frankfurt, Frankfurt am Main, Germany

2 Cancer Prognostics and Health Outcomes Unit, Division of Urology, University of Montréal Health Center, Montréal, Québec, Canada

3 Martini-Klinik Prostate Cancer Center, University Hospital Hamburg-Eppendorf, Hamburg, Germany

4 Department of Urology, University of Naples Federico II, Naples, Italy

5 Department of Urology and Division of Experimental Oncology, URI, Urological Research Institute, IBCAS San Raffaele Scientific Institute, Milan, Italy

6 Department of Urology, Comprehensive Cancer Center, Medical University of Vienna, Vienna, Austria

7 Institute for Urology and Reproductive Health, I.M. Sechenov First Moscow State Medical University, Moscow, Russia

8 Department of Urology, University of Jordan, Amman, Jordan

\section{Introduction}

Bladder cancer (BCa) stage and grade is strongly associated with cancer-specific mortality (CSM), therefore early diagnosis and treatment are imperative $[1,2]$. Patients in rural areas may have more limited access to care and thus may be at risk for delayed bladder cancer diagnosis and treatment [3]. Many, predominantly historical, studies suggested that mortality rates were higher in rural areas compared to urban $[4,5]$. To study that concept, we applied the official definition of rural and urban areas in the United States (US) according to the US Census Bureau and stratified our analyses according to urbanized areas (UA, $\geq 50,000$ inhabitants) vs. urban clusters (UC, 2500-50,000 inhabitants) vs. rural areas (RA <2500 inhabitants) [6]. Within this stratification, we assessed potential differences in $\mathrm{BCa}$ stage at presentation, treatment patterns and cancer-specific mortality (CSM), as well as other cause mortality (OCM) in the most contemporary version of the SEER database. We 
hypothesized that RA residency status will be associated with higher stage at diagnosis and higher CSM, even after strict adjustment for OCM.

\section{Materials and methods}

\section{Study population}

The current SEER database samples $34.6 \%$ of the US population and approximates it in demographic composition and cancer incidence [7]. Within the SEER database (2004-2016), we identified patients $\geq 18$ years old with histologically confirmed bladder cancer (International Classification of Disease for Oncology [ICD-O] site code C67.0-67.9). Cases identified only at autopsy or death certificate, were excluded. According to US Census Bureau definition, we included two types of metropolitan areas: urban areas (UA) and urban clusters (UC). UA were defined as areas with 50,000 or more inhabitants. UC were defined as areas with at least 2500 but fewer than 50,000 inhabitants. Rural areas (RA), conversely, were defined as all population, housing, and territory not included within UA or UC.

\section{Statistical analyses}

Covariates in multivariate logistic regression analyses consisted of age at diagnosis, sex, race, grade, T-stage and $\mathrm{N}$-stage and $\mathrm{M}$-stage. Cumulative incidence plots assessed cancer-specific mortality (CSM), as well as other cause mortality (OCM) according to residency status (RA vs. UC vs. UA) in the overall cohort and in stage-specific analyses. In stage-specific, fully propensity score (PS)-adjusted analyses, two comparisons were made: (1) RA vs. UA, (2) UC vs. UA. For each individual comparison, PS-adjustment was applied for age, sex and socioeconomic status. Additional multivariate adjustment in competing risks regression (CRR) models was applied for age at diagnosis, sex, race, grade, surgical treatment type and chemotherapy [8]. Moreover, in CRR models, CSM estimates were adjusted for OCM and vice versa. In all statistical analyses, $\mathrm{R}$ software environment for statistical computing and graphics ( $\mathrm{R}$ version 3.6.1) was used. All tests were two-sided with a level of significance set at $p<0.05$.

\section{Results}

\section{Descriptive characteristics of the study population}

Within the SEER database, 222,330 bladder cancer patients of all stages were identified (Table 1). Of these, 3496 (1.6\%) resided in RA, 25,462 (11.5\%) resided in UC and 193,372 (87\%) resided in UA. Mean age was comparable between RA, UC, and UA (72 vs. 72 vs. 73 years). High socioeconomic status was more prevalent in UC $(35 \%)$ vs. RA $(28.6 \%)$ vs. UA (23.4\%). Married patients were most frequently recorded in RA (62.1\%) followed by UC $(60.5 \%)$ followed by UA ( $58.3 \%)$. Caucasian race was most frequently recorded in RA (96.6\%) followed by UC $(93.7 \%)$ followed by UA $(88.3 \%)$.

\section{Stage at presentation and treatment rates}

Tumor stage was comparable and absolute differences ranged from 0 to $1.2 \%$ (Table 1). Similarly, in RA vs. UC vs. UA, N0-stage was recorded in $93 \%$ vs. $93.0 \%$ vs. $92.5 \%$ and M0-stage was recorded in $94.1 \%$, vs. $94.4 \%$ vs. $94.2 \%$, respectively. Conversely, low tumor grade was more prevalent in RA (39.6\%) vs. UC (38.8\%) vs. UA (34.6\%).

Rates of radical cystectomy were virtually the same: RA (9.8\%), vs. UC (9.1\%) vs. UA (9.1\%). Conversely, chemotherapy use was marginally more frequently recorded in UC (23.6\%) vs. RA (22.1\%) vs. UA (20.5\%).

\section{Logistic regression predicting advanced stage or treatment}

In all six separate multivariate logistic regression analyses, predicting 1) advanced tumor stage $\left.\left(\mathrm{T}_{3-4}\right), 2\right)$ node positive stage $\left.\left(\mathrm{N}_{1-3}\right), 3\right)$ metastatic stage $\left.\left(\mathrm{M}_{1}\right), 4\right)$ high grade differentiation, 5) treatment with RC and 6) chemotherapy use, RA residency status did not predict the examined outcome (Table 2). Conversely, UC residency status was protective (OR 0.96, $p=0.003$ ) from high grade differentiation and from node positive stage (OR $0.90, p=0.03$ ), when referenced to UA residency status. Moreover, UC residency status predicted higher rates of chemotherapy treatment (OR $1.21, p<0.001)$, when referenced to UA residency status.

\section{Cumulative incidence plots of cancer-specific and other cause mortality}

In the overall analyses, that included all tumor stages (Fig. 1), 10-year CSM rates according to RA vs. UC vs. UA status were $20.0 \%$ vs. $20.1 \%$ vs. $18.8 \%(p<0.001)$. In stagespecific analyses, 10 -year CSM rates were $12.2 \%$ vs. $11.6 \%$ vs. $10.7 \%(p=0.001)$ in stage T1 BCa patients, according to respectively RA vs. UC vs. UA status. Conversely, in all other stage-specific analyses, no significant differences in CSM were recorded, according to RA vs. UC vs. UA residency status (data not shown).

In the overall analyses, 10-year OCM rates according to RA vs. UC vs. UA status were $30.9 \%$ vs. $27.7 \%$ vs. 
Table 1 Patient and tumor characteristics of 222,330 bladder cancer patients of all stages, stratified according to rural or urban residency status, diagnosed within the SEER database from 2004 to 2016

\begin{tabular}{|c|c|c|c|c|c|}
\hline & Cat/Stat & Overall 222,330 & Rural Areas 3496 (1.6) & $\begin{array}{l}\text { Urban Clusters } \\
25,462(11.5)\end{array}$ & $\begin{array}{l}\text { Urbanized } \\
\text { Areas 193,372 } \\
(87)\end{array}$ \\
\hline Age at diagnosis & Mean (STE) & $71.4(0.025)$ & $70.7(0.192)$ & $70.9(0.073)$ & $71.5(0.027)$ \\
\hline Age at diagnosis & Median (IQR) & $72(64-80)$ & $72(64-79)$ & $72(63-79)$ & $73(64-81)$ \\
\hline \multirow[t]{2}{*}{ Sex } & Female & $53,379(24)$ & $777(22.2)$ & $5649(22.2)$ & $46,953(24.3)$ \\
\hline & Male & $168,951(76)$ & $2719(77.8)$ & $19,813(77.8)$ & $146,419(75.7)$ \\
\hline \multirow[t]{3}{*}{ Race } & White & $197,907(89)$ & $3376(96.6)$ & $23,848(93.7)$ & $170,683(88.3)$ \\
\hline & Black & $12,489(5.6)$ & $92(2.6)$ & $960(3.8)$ & $11,437(5.9)$ \\
\hline & Other & $11,934(5.4)$ & $28(0.8)$ & $654(2.6)$ & $11,252(5.8)$ \\
\hline \multirow[t]{2}{*}{ Socio economic status } & 1 quartile & $55,288(24.9)$ & $1000(28.6)$ & $8993(35.3)$ & $45,295(23.4)$ \\
\hline & 2-3-4 quartile & $167,042(75.1)$ & $2496(71.4)$ & $16,469(64.7)$ & $148,077(76.6)$ \\
\hline \multirow[t]{5}{*}{ Marital status } & Married & $130,378(58.6)$ & $2171(62.1)$ & $15,400(60.5)$ & $112,807(58.3)$ \\
\hline & Never Married & $22,731(10.2)$ & $255(7.3)$ & $2166(8.5)$ & $20,310(10.5)$ \\
\hline & Separated/ Divorced & $18,531(8.3)$ & $306(8.8)$ & $2286(9)$ & $15,939(8.2)$ \\
\hline & Unknown & $18,103(8.1)$ & $276(7.9)$ & $1817(7.1)$ & $16,010(8.3)$ \\
\hline & Widowed & $32,587(14.7)$ & $488(14)$ & $3793(14.9)$ & $28,306(14.6)$ \\
\hline \multirow[t]{4}{*}{ Insurance status } & Medicaid insured & $12,848(5.8)$ & $298(8.5)$ & $1704(6.7)$ & $10,846(5.6)$ \\
\hline & Insured other & $148,260(66.7)$ & $2243(64.2)$ & $16,780(65.9)$ & $129,237(66.8)$ \\
\hline & Uninsured & $2673(1.2)$ & $55(1.6)$ & 417 (1.6) & $2201(1.1)$ \\
\hline & Unknown & $58,549(26.3)$ & $900(25.7)$ & $6561(25.8)$ & $51,088(26.4)$ \\
\hline \multirow[t]{4}{*}{ T-stage } & $<=\mathrm{T} 1$ & $173,890(78.2)$ & $2697(77.1)$ & $19,729(77.5)$ & $151,464(78.3)$ \\
\hline & $\mathrm{T} 2$ & $31,465(14.2)$ & $530(15.2)$ & 3797 (14.9) & $27,138(14)$ \\
\hline & $\mathrm{T} 3$ & 8679 (3.9) & $139(4)$ & $937(3.7)$ & 7603 (3.9) \\
\hline & $\mathrm{T} 4$ & $8296(3.7)$ & $130(3.7)$ & 999 (3.9) & 7167 (3.7) \\
\hline \multirow[t]{5}{*}{$\mathrm{N}$-stage } & No & $205,748(92.5)$ & 3264 (93.4) & $23,670(93)$ & $178,814(92.5)$ \\
\hline & N1 & $4338(2)$ & $55(1.6)$ & $463(1.8)$ & $3820(2)$ \\
\hline & $\mathrm{N} 2$ & 3807 (1.7) & $58(1.7)$ & $434(1.7)$ & 3315 (1.7) \\
\hline & $\mathrm{N} 3$ & $807(0.4)$ & $12(0.3)$ & $88(0.3)$ & $707(0.4)$ \\
\hline & $\mathrm{Nx}$ & $7630(3.4)$ & $107(3.1)$ & 807 (3.2) & $6716(3.5)$ \\
\hline \multirow[t]{3}{*}{ M-stage } & M0 & $209,506(94.2)$ & $3291(94.1)$ & $24,040(94.4)$ & $182,175(94.2)$ \\
\hline & M1 & 7887 (3.5) & $130(3.7)$ & $897(3.5)$ & $6860(3.5)$ \\
\hline & MX & $4937(2.2)$ & $75(2.1)$ & $525(2.1)$ & $4337(2.2)$ \\
\hline \multirow[t]{3}{*}{ Grade } & $\mathrm{G} 1 / \mathrm{G} 2$ & $78,240(35.2)$ & 1383 (39.6) & 9873 (38.8) & $66,984(34.6)$ \\
\hline & G3/G4 & $105,459(47.4)$ & $1625(46.5)$ & $11,948(46.9)$ & $91,886(47.5)$ \\
\hline & Unknown & $38,631(17.4)$ & $488(14)$ & $3641(14.3)$ & $34,502(17.8)$ \\
\hline \multirow[t]{5}{*}{ Type of surgery } & None & $14,347(6.5)$ & $233(6.7)$ & $1786(7)$ & $12,328(6.4)$ \\
\hline & Partial cystectomy & $2779(1.2)$ & $39(1.1)$ & $317(1.2)$ & $2423(1.3)$ \\
\hline & Radical cystectomy & $20,353(9.2)$ & $343(9.8)$ & $2317(9.1)$ & $17,693(9.1)$ \\
\hline & TURB & $183,607(82.6)$ & $2843(81.3)$ & $20,813(81.7)$ & $159,951(82.7)$ \\
\hline & Unknown & $1244(0.6)$ & $38(1.1)$ & $229(0.9)$ & $977(0.5)$ \\
\hline \multirow[t]{2}{*}{ Chemotherapy } & No/Unknown & $175,963(79.1)$ & $2725(77.9)$ & $19,451(76.4)$ & $153,787(79.5)$ \\
\hline & Yes & 46,367 (20.9) & $771(22.1)$ & $6011(23.6)$ & $39,585(20.5)$ \\
\hline
\end{tabular}

$25.6 \%(p<0.01)$. Similarly, 10-year OCM rates in stage T1 BCa patients were $33.4 \%$ vs. $29.3 \%$ vs. $27.1 \%$ (p < 0.01 ), according to respectively RA vs. UC vs. UA residency status. Conversely, in all other stage-specific analyses, no significant differences in OCM were recorded, according to RA vs. UC vs. UA residency status (data not shown). 
Table 2 Six separate logistic regression models predicting advanced tumor stage (T3-4, N1-3 or M1) high grade differentiation or treatment with radical cystectomy $(\mathrm{RC})$ or chemotherapy according to residency status

\begin{tabular}{|c|c|c|c|c|c|c|}
\hline & \multicolumn{2}{|c|}{$\begin{array}{l}\text { A. Predictors of advanced } \\
\text { T-stage }\left(\mathrm{T}_{3-4}\right)\end{array}$} & \multicolumn{2}{|c|}{$\begin{array}{l}\text { B. Predictors of node positive stage } \\
\left(\mathrm{N}_{1-3}\right)\end{array}$} & \multicolumn{2}{|c|}{$\begin{array}{l}\text { C. Predictors of metastatic stage } \\
\mathrm{M}_{1}\end{array}$} \\
\hline & OR $(95 \% \mathrm{CI})$ & $\mathrm{p}$ value & OR $(95 \% \mathrm{CI})$ & $\mathrm{p}$ value & OR $(95 \% \mathrm{CI})$ & $\mathrm{p}$ value \\
\hline Urbanized Areas (Ref.) & 1 & - & 1 & - & 1 & - \\
\hline Rural Areas & $1.09(0.94-1.25)$ & 0.25 & $0.81(0.62-1.04)$ & 0.10 & $1.14(0.93-1.38)$ & 0.19 \\
\hline \multirow[t]{3}{*}{ Urban Clusters } & $1.03(0.98-1.09)$ & 0.25 & $0.90(0.82-0.99)$ & $\mathbf{0 . 0 3}$ & $1.03(0.94-1.11)$ & 0.49 \\
\hline & \multicolumn{2}{|c|}{$\begin{array}{l}\text { D. Predictors of high grade differen- } \\
\text { tiation }\end{array}$} & \multicolumn{2}{|c|}{ E. Predictors of treatment with RC } & \multicolumn{2}{|c|}{$\begin{array}{l}\text { F. Predictors of treatment with } \\
\text { chemotherapy }\end{array}$} \\
\hline & OR $(95 \% \mathrm{CI})$ & $\mathrm{p}$ value & OR $(95 \% \mathrm{CI})$ & $\mathrm{p}$ value & OR $(95 \% \mathrm{CI})$ & $\mathrm{p}$ value \\
\hline Urbanized Areas (Ref.) & 1 & - & 1 & - & 1 & - \\
\hline Rural Areas & $0.94(0.87-1.01)$ & 0.10 & $1.01(0.87-1.17)$ & 0.87 & $1.09(0.99-1.18)$ & 0.051 \\
\hline Urban Clusters & $0.96(0.93-0.99)$ & 0.009 & $0.94(0.89-1.00)$ & 0.055 & $1.21(1.17-1.25)$ & $<0.001$ \\
\hline
\end{tabular}

p-values below 0.05 are displayed in bold

Fig. 1 Cumulative incidence plots depicting cancer-specific mortality (CSM) and other cause mortality (OCM) according to residency status (rural area [RA] vs. urban cluster [UC] vs. urbanized area [UA]) in overall bladder cancer $(\mathrm{BCa})$ patients

\section{CSM- and OCM-rates according to rural vs. urban status in all $\mathrm{BCa}$ patients}

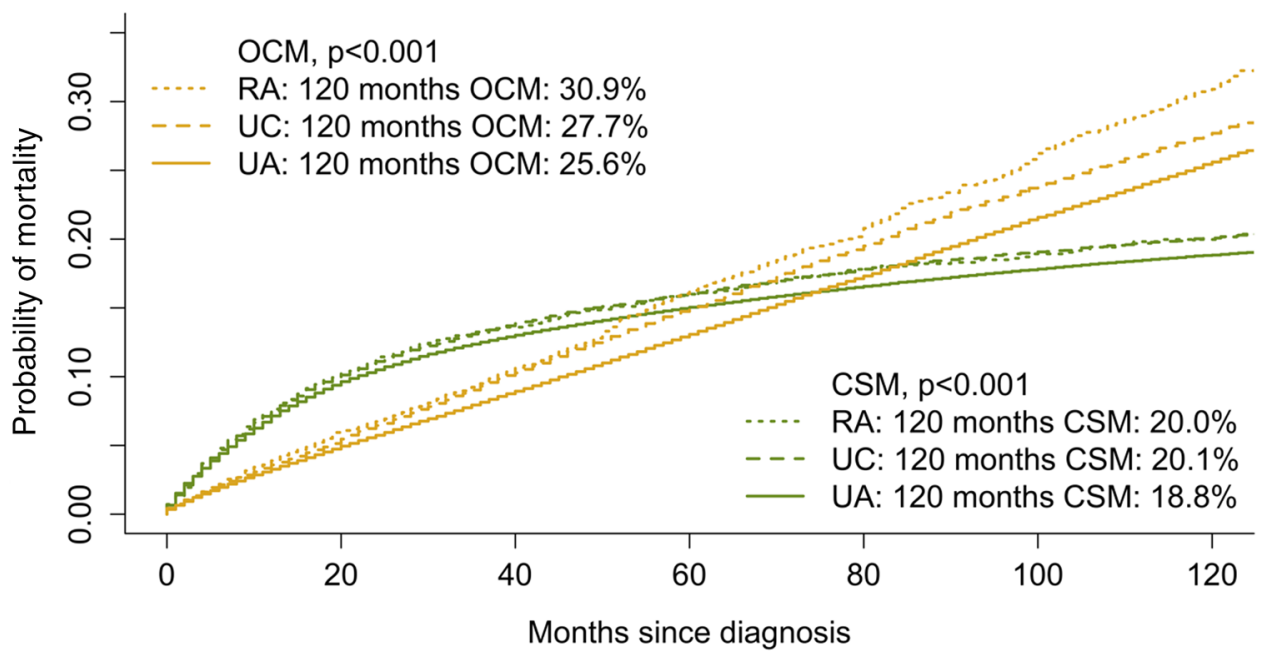

\section{Matched and multivariate competing risks regression analyses}

\section{(A) Cancer-specific mortality}

After PS-matching and additional multivariate adjustments, CSM differences were recorded between 1) RA vs. UA and 2) UC vs. UA stage T1 BCa patients. Specifically, 10-year CSM rates were $12.2 \%$ vs. $11.1 \%(p=0.09)$, according to RA vs. UA and $11.6 \%$ vs. $10.5 \%(p<0.001)$, according to UC vs. UA residency status, respectively (Fig. 2). When referenced to UA in CRR (Table 3), RA and UC residency status were a risk factor for higher CSM in stage $\mathrm{T} 1 \mathrm{BCa}$ (HR 1.21, $p=0.02$ and 1.17, $p<0.001$ ).
All other PS-matched and multivariate analyses, did not reveal statistically significant differences in CSM.

(B) Other cause mortality

After PS-matching and additional multivariate adjustments, OCM differences were also recorded between 1) RA vs. UA and 2) UC vs. UA stage T1 BCa patients. Specifically, 10 -year OCM rates were $33.4 \%$ vs. $26.5 \%(p<0.001)$ according to RA vs. UA and $29.3 \%$ vs. $26.6 \%$ (p<0.001), according to UC vs. UA residency status, respectively (Fig. 2). When referenced to UA in CRR (Table 3), RA and UC residency status were a risk factor for higher OCM in stage T1 BCa (HR 1.29 and 1.18, both $p<0.001$ ). All other 


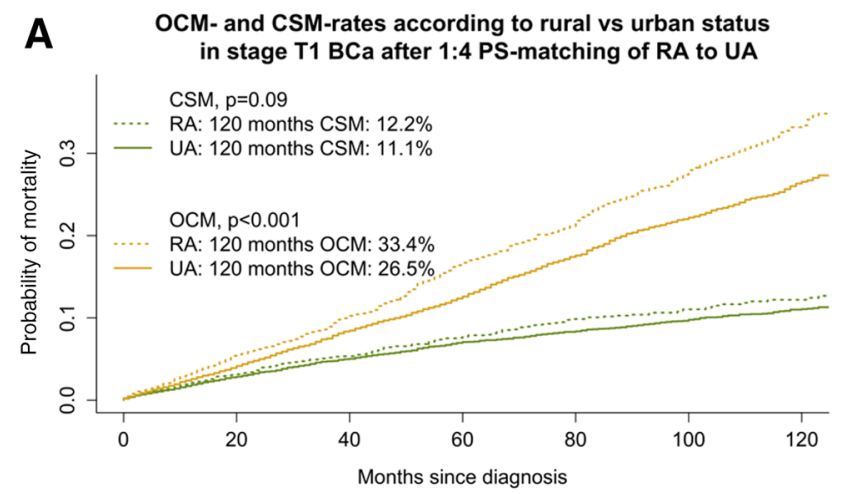

Fig. 2 Cumulative incidence plots after 1:4 matching of (a) rural area (RA) residency status $(n=2,651 \mathrm{RA})$ with urbanized area (UA) residency status ( $\mathrm{n}=10,604 \mathrm{UC})$, or of (b) urban cluster (UC) residency

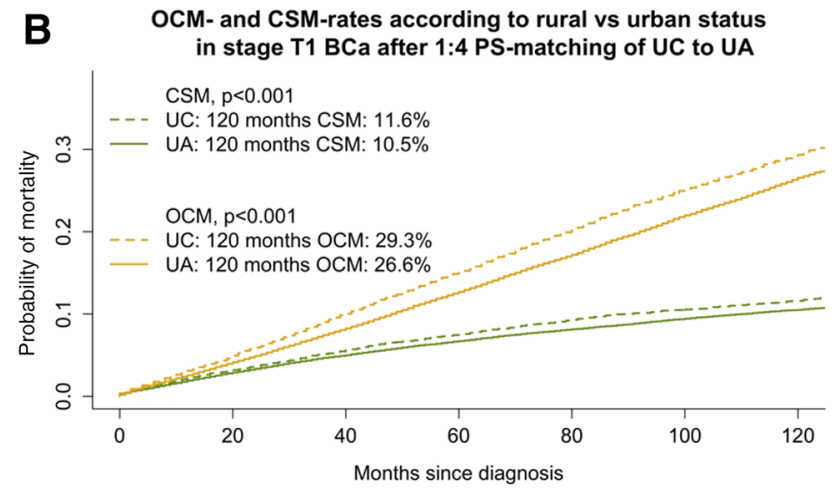

status ( $n=19,437$ UC) with UA $(n=77,748)$, depicting cancer-specific mortality (CSM) and other cause mortality (OCM) in stage T1N0M0 BCa patients
Table 3 Two separate competing risks regression analyses, after matching of A) rural areas with urban areas and B) urbanized clusters with urban areas. Multivariate adjustment was made for Age at diag- nosis, tumor grade, sex, race, socioeconomic status, surgical treatment and chemotherapy use

\begin{tabular}{|c|c|c|c|c|c|c|c|c|}
\hline & \multicolumn{4}{|l|}{ CSM } & \multicolumn{4}{|l|}{ OCM } \\
\hline & HR univariate & $\mathrm{p}$ value & HR multivariate & $\mathrm{p}$ value & HR univariate & $\mathrm{p}$ value & HR multivariate & $\mathrm{p}$ value \\
\hline \multicolumn{9}{|c|}{ A) Matched RA with UA } \\
\hline UA & 1.00 (Ref.) & - & 1.00 (Ref.) & - & 1.00 (Ref.) & - & 1.00 (Ref.) & - \\
\hline RA & $1.14(0.98-1.33)$ & 0.09 & $1.21(1.03-1.41)$ & 0.019 & $1.28(1.16-1.42)$ & $<0.001$ & $1.29(1.17-1.43)$ & $<0.001$ \\
\hline \multicolumn{9}{|c|}{ B) Matched UA with UC } \\
\hline UA & 1.00 (Ref.) & - & 1.00 (Ref.) & - & 1.00 (Ref.) & - & 1.00 (Ref.) & - \\
\hline UC & $1.13(1.07-1.2)$ & $<0.001$ & $1.17(1.1-1.24)$ & $<0.001$ & $1.17(1.12-1.22)$ & $<0.001$ & $1.18(1.14-1.23)$ & $<0.001$ \\
\hline
\end{tabular}

PS-matched and multivariate analyses, did not reveal statistically significant differences in OCM.

\section{Discussion}

We applied the official definition of rural and urban areas according to the US Census Bureau and stratified our analyses according to three different types of residential areas. Our work revealed several important observations.

First of all BCa patients in our analysis, only $1.6 \%$ accounted for RA residency status. Conversely, $11.5 \%$ were recorded in UC and $87 \%$ in UA residential areas, respectively. These rates differ substantially from the officially reported composition of the US according to the US Census Bureau [6]: In the year 2010, there were 486 UA and 3087 UC in the United States. UA accounted for $71.2 \%$ of the US population, while $9.5 \%$ resided in UC. Conversely, $19.3 \%$ resided in RA. Taking into account these major differences in the composition of the US population, the SEER database does not reflect the US in terms of urban vs. rural residency status. Rural regions of the US population are underrepresented in the SEER database. This fact is attributable to the composition of the SEER registries, that encompass mainly metropolitan regions and as such a majority of patients from UA or UC are registered in the database [9]. In consequence, it is difficult to analyze the effect of rural residency status within the SEER database, due to small numbers of RA observations. Ideally, future iterations of the SEER database should oversample rural areas, to better reflect the rural composition of the US.

Second despite the relatively small proportion of patients from rural areas, the size of the SEER database, allowed us to make important observations. RA residency status was not associated with meaningful differences in stage at presentation or with inferior access to care. This is conflicting with two previous reports from Monroe et al. (review from 1992) [10] and Hashibe et al. ( $n=32,498$ metropolitan and $4906(13.1 \%)$ rural inhabitants, from 2014) [3]. Both investigators reported that rural cancer patients were diagnosed at a higher stage.

However, in our report, UC residency status was protective against $\mathrm{N}_{1-3}$ stage at presentation (HR: 0.90) and high grade differentiation (HR 0.96) compared to UA. Interestingly, chemotherapy rates were higher in UC than in UA 
(HR 1.21). To the best of our knowledge, no previous report examined differences in stage, treatment and mortality, according to substages of urban differentiation (UC vs. UA) and thus, we cannot compare our results to other reports.

Third in multivariately adjusted and matched analyses, we only observed marginal CSM differences (absolute difference of 1.2\% in 10-year CSM rates: 20 vs. $18.8 \%$ ) between RA and UA residency status. This is in agreement with our results regarding stage and grade distribution in rural patients, that were not worse for RA than UA patients. Similarly, patients residing within UC only exhibited marginal differences in CSM in comparison to UA residency status (absolute difference of 1.3\% in 10-year CSM rates: $20.1 \%$ vs. $18.8 \%$ ). Due to the large sample size of the SEER database, these marginal differences resulted in a statistically significant difference. Thus, prior works, that found higher CSM in RA [3-5] were numerically confirmed in our analyses. Nevertheless, it has to be emphasized that despite the statistical significance of our findings, the absolute differences in CSM are of unknown clinical importance. Moreover, in stage-specific analyses, this difference was only recorded in stage T1 patients. In higher tumor stages, no significant CSM differences between RA and UC or UA were registered. In consequence, rural area residency status predisposes to marginally higher CSM rates, but only in stage T1N0M0 patients. The overall result of no CSM difference is in concordance with three other studies [11-13]. Specifically, two of these studies focused on $\mathrm{BCa}$ and examined complications of radical cystectomy patients according to urban vs. rural status in a homogenous group of patients with the same insurance [11], as well as guideline adherence rates and mortality in nonmuscle invasive $\mathrm{BCa}$ patients in a rural state [12]. However, no previous study examined CSM in stage-specific fashion. Therefore, we cannot compare our results of CSM in T1N0M0 patients with other reports. In consequence, more studies of CSM in RA BCa patients are warranted to further evaluate this potentially worrisome signal.

In the final part of our analyses we focused on OCM in RA vs. UC vs. UA patients of all stages. OCM rates demonstrated significant and meaningful differences according to residence in RA vs. UC vs. UA. Specifically, 10-year OCM rates were highest in RA (30.9\%), followed by UC (27.7\%) and UA $(25.6 \%)$. The differences persisted and remained highly statistically significant, even after PS-matching for age, sex and socioeconomic status, as well as after multivariate adjustment for residual confounders and for the effect of competing cancer-specific mortality in stage T1NOM0 BCa patients: RA $33.4 \%$, followed by UC $29.3 \%$ and UA $26.5 \%$, but to a much lesser extent in all other BCa stages. In consequence, the observed OCM disadvantage in rural patients and to a lesser, albeit important and significant extent in UC patients, is worrisome. It indicates worse general health of rural area and urban cluster inhabitants with $\mathrm{T} 1 \mathrm{BCa}$. It suggests higher prevalence of comorbidities, that are directly associated with OCM. Such OCM disadvantage in T1 RA and UC BCa patients warrants consideration, when treatments with important morbidity are considered. Radical cystectomy and chemotherapy for $\mathrm{BCa}$ represent such treatments. However, since our report represents the first signal suggesting an OCM disadvantage in RA and UC T1NOM0 $\mathrm{BCa}$ patients, further studies are clearly needed to validate or refute this observation.

Taken together our observations indicate that patients residing in rural areas, are not diagnosed at a later stage or with higher BCa grade. Moreover, they appear to benefit of available treatments, at least at the same rate as their urban counterparts. However, we recorded important and statistically significant OCM and marginal but statistically significant CSM differences, that were operational in stage T1N0M0 RA and UC patients, but not in other stage-specific analyses. OCM rates were highest in RA and were followed by UC and UA. The OCM differences potentially indicate worse general health of rural and urban cluster T1 BCa patients. Moreover, CSM differences suggest a marginal, albeit detectable disadvantage for RA and UC T1NOM0 BCa patients, relative to their UA counterparts. Further validation studies of CSM and OCM in RA and UC vs. UA BCa patients are clearly warranted. The accuracy of these observations may be limited by the relative paucity of rural patient composition, within the SEER database, and most likely within all other databases as well.

Despite multiple novel and important observations, several limitations may be applicable to our study. First and foremost, the number of patients with RA residency status was low and did not allow a proportional representation of the composition of the US population. Moreover, the retrospective, population-based nature of the SEER database did not allow us to control for some unavailable covariates and comorbidities. However, we adjusted all our analyses for OCM, which is a marker for the most important comorbidities, namely those resulting in death from other causes. Nevertheless, it would be of great interest to explore baseline comorbidity status in RA vs. UC vs. UA, that could potentially better explain the OCM disadvantage in RA vs. UC vs. UA. Limitations related to the retrospective, populationbased nature of the SEER database, apply to this, as well as to other similar analyses that were based on the SEER database or on other similar large scale data repositories, such as National Cancer Data Base, National Inpatient Sample or National Surgical Quality Improvement Program.

\section{Conclusion}

We did not observe meaningful differences in access to treatment or stage distribution, according to residency status. However, RA and to a lesser extent UC residency status, 
were associated with higher OCM and marginally higher CSM in T1N0M0 patients. This observation should be further validated or refuted in additional epidemiological investigations, that focus on RA and UC residents.

\begin{abstract}
Authors' contributions MD: Conceptualization; Data curation; Formal analysis; Investigation; Methodology; Validation; Visualization; Roles/ Writing —original draft. LFS: Data curation; Formal analysis; Investigation; Methodology; Roles/Writing — original draft. CCR: Data curation; Formal analysis; Investigation; Methodology; Roles/Writing—original draft. LN: Data curation; Formal analysis; Investigation; Methodology; Roles/Writing — original draft. ZT: Data curation; Formal analysis; Investigation; Methodology; Software; Resources. FCR: Resources; Supervision; Validation; Writing-review \& editing. AB: Resources; Supervision; Validation; Writing - review \& editing. LAK: Resources; Supervision; Validation; Writing—review \& editing. DT: Resources; Supervision; Validation; Writing — review \& editing. SFS: Resources; Supervision; Validation; Writing — review \& editing. FS: Resources; Supervision; Validation; Writing - review \& editing. FKHC: Resources; Supervision; Validation; Writing — review \& editing; Project administration; Supervision. PIK: Resources; Supervision; Validation; Writingreview \& editing; Project administration; Supervision.
\end{abstract}

Funding Open Access funding enabled and organized by Projekt DEAL.

Data availability SEER data are publicly available. Access to the SEER database is provided for researchers by the National Health Institute.

Code availability Custom code upon request.

\section{Compliance with ethical standards}

Conflict of interest Our research was conducted in the absence of any commercial or financial relationships that could be construed as a potential conflict of interest. The authors declare that they have no known competing financial interests or personal relationships that could have appeared to influence the work reported in this paper.

Ethical approval Approval was waived by the local ethics committee, as SEER data are publicly available and de-identified.

Open Access This article is licensed under a Creative Commons Attribution 4.0 International License, which permits use, sharing, adaptation, distribution and reproduction in any medium or format, as long as you give appropriate credit to the original author(s) and the source, provide a link to the Creative Commons licence, and indicate if changes were made. The images or other third party material in this article are included in the article's Creative Commons licence, unless indicated otherwise in a credit line to the material. If material is not included in the article's Creative Commons licence and your intended use is not permitted by statutory regulation or exceeds the permitted use, you will need to obtain permission directly from the copyright holder. To view a copy of this licence, visit http://creativecommons.org/licenses/by/4.0/.

\section{References}

1. Soukup V, Capoun O, Cohen D, Hernandez V, Babjuk M, Burger M, Comperat E, Gontero P, Lam T, MacLennan S, Mostafid
AH, Palou J, van Rhijn BWG, Roupret M, Shariat SF, Sylvester R, Yuan Y, Zigeuner R (2017) Prognostic Performance and Reproducibility of the 1973 and 2004/2016 World Health Organization Grading Classification Systems in Non-muscle-invasive Bladder Cancer: A European Association of Urology Non-muscle Invasive Bladder Cancer Guidelines Panel Systematic Review. Eur Urol 72(5):801-813. https:// doi.org/10.1016/j.eururo.2017.04.015

2. van Rhijn BW, van der Kwast TH, Alkhateeb SS, Fleshner NE, van Leenders GJ, Bostrom PJ, van der Aa MN, Kakiashvili DM, Bangma CH, Jewett MA, Zlotta AR (2012) A new and highly prognostic system to discern T1 bladder cancer substage. Eur Urol 61(2):378-384. https://doi.org/10.1016/j.eururo.2011.10.026

3. Hashibe M, Kirchhoff AC, Kepka D, Kim J, Millar M, Sweeney C, Herget K, Monroe M, Henry NL, Lopez AM, Mooney K (2018) Disparities in cancer survival and incidence by metropolitan versus rural residence in Utah. Cancer Med 7(4):1490-1497. https://doi.org/10.1002/cam4.1382

4. Wilkinson D, Cameron K (2004) Cancer and cancer risk in South Australia: what evidence for a rural-urban health differential? Aust J Rural Health 12(2):61-66. https://doi.org/10 $.1111 / \mathrm{j} .1038-5282.2004 .00555 . x$

5. Singh GK, Siahpush M, Williams SD (2012) Changing urbanization patterns in US lung cancer mortality, 1950-2007. J Community Health 37(2):412-420. https://doi.org/10.1007/ s10900-011-9458-3

6. Ratcliffe M, Burd C, Holder K, Fields A (2016) Defining Rural at the U.S. Census Bureau. ACSGEO-1, US Census Bureau, Washington, DC, 2016 https://www2.census.gov/geo/pdfs/refer ence/ua/Defining_Rural.pdf. Retrieved 2nd of April.2020

7. Howlader N, Noone A, Krapcho M, Miller D, Brest A, Yu M, Ruhl J, Tatalovich Z, Mariotto A, Lewis D, Chen H, Feuer E, Cronin K (2018) SEER Cancer Statistics Review, 1975-2016, National Cancer Institute. Bethesda, MD. based on November 2018 SEER data submission, posted to the SEER web site, April 2019. doi:https://seer.cancer.gov/csr/1975_2016/

8. Lau B, Cole SR, Gange SJ (2009) Competing Risk Regression Models for Epidemiologic Data. Am J Epidemiol 170(2):244 256. https://doi.org/10.1093/aje/kwp107

9. About the SEER Registries. National Cancer Institute, Surveillance, Epidemiology, and End Results (SEER) Program https:// seer.cancer.gov/registries/list.html. Retrieved 7th April, 2020

10. Monroe AC, Ricketts TC, Savitz LA (1992) Cancer in rural versus urban populations: a review. J Rural Health 8(3):212220. https://doi.org/10.1111/j.1748-0361.1992.tb00354.x

11. Sadowski DJ, Warner H, Scaife S, McVary KT, Alanee SR (2018) 30-day all-cause hospital readmission after cystectomy: no worse for rural Medicare residents. Urol Oncol 36(3):89. e87-89.e11. https://doi.org/10.1016/j.urolonc.2017.11.013

12. Tobert CM, Nepple KG, McDowell BD, Charlton ME, Mott SL, Gruca TS, Quast L, Erickson BA (2019) Compliance With American Urological Association Guidelines for Nonmuscle Invasive Bladder Cancer Remains Poor: Assessing Factors Associated With Noncompliance and Survival in a Rural State. Urology 132:150-155. https://doi.org/10.1016/j.urolo gy.2019.06.021

13. Riaz SP, Horton M, Kang J, Mak V, Luchtenborg M, Moller H (2011) Lung cancer incidence and survival in England: an analysis by socioeconomic deprivation and urbanization. J Thorac Oncol 6(12):2005-2010. https://doi.org/10.1097/ JTO.0b013e31822b02db

Publisher's Note Springer Nature remains neutral with regard to jurisdictional claims in published maps and institutional affiliations. 